\title{
Immediately Effects of Static Stretching of the Ankle Plantar Flexor for 5 Minutes on Balance Control and Muscle Activity in Healthy Young Adults
}

\author{
Sang-Hyuk Yoon, Jae-Won Lee, Dongyeop Lee, Ji-Heon Hong, Jae-Ho Yu, Jin-Seop Kim, Seong-gil Kim \\ Department of Physical Therapy, College of Health Science, Sunmoon University, Asan, Republic of Korea
}

\begin{abstract}
Purpose: The purpose of this study is to confirm the effect of static stretching of the plantar flexor for 5 minutes on balance and ankle muscle activity when walking in young adults.

Methods: This study experimented on 20 healthy college students without vestibular and musculoskeletal diseases. Subjects performed static stretching intervention of plantar flexor for 5 minutes on a stretch board set at $15^{\circ}$ to $25^{\circ}$ Balance was measured four times before intervention (pre), after intervention (post), 5 minutes after intervention (post $5 \mathrm{~min}$ ), 10 minutes after intervention (post $10 \mathrm{~min}$ ), and ankle muscle activity was measured during walking. For the analysis and post hoc analysis, one-way Repeated Measure ANOVA and Fisher's LSD (Last Significant Difference) was performed to find out the change in balance and the activity of ankle muscles before static stretching, pre, post, post 5 minutes rest, post 10 minutes rest.

Results: There was no significant difference in weight distribution index (WDI) in balance, but stability index (ST) showed a significant difference, and there was also a significant difference in correlation pre, post, post $5 \mathrm{~min}$ rest, post 10 minutes rest $(p<0.05)$. There was no significant difference in ankle muscle activity during walking in Tibialis anterior (TA), Medial gastrocnemius (GM), and Lateral gastrocnemius (GL) ( $p>0.05)$.
\end{abstract}

Conclusion: The stability index (ST) increased significantly immediately after static stretching and decreased after 5 minutes. After static stretching, at least 5 minutes of rest are required to restore balance.

Keywords: Static stretching, Postural sway, Plantar flexor, Static balance, Tibialis anterior, Gastrocnemius

\section{INTRODUCTION}

Walking is an essential activity of everyday life and the basis of all sports movements. Walking is regarded as one of the simplest physical activities, but actually, it is a complex activity that requires comprehensive integration between the musculoskeletal and nervous systems. ${ }^{1}$ Standing on both feet before walking is inevitably unstable because the body's center of gravity is located higher than the relatively narrow basal plane. However, since most of the functional activities performed by humans are performed in a standing position, research on maintaining balance in a standing position has been conducted for many years. Studies to date have shown that humans require ankle and hip joint strategies to stabilize the body during standing and that ankle joints contribute much to balance., ${ }^{2,3}$

Received Nov 16, 2021 Revised Dec 15, 2021

Accepted Dec 15, 2021

Corresponding author Seong-gil Kim

E-mail sungkil83@naver.com
Appropriate ankle strategies require passive torque through muscle tissue tension, stiffness of tissues around joints such as ligaments or tendons, voluntary body movements due to external effects, and active torque directed by central nervous system signals to contract and regulate muscles. ${ }^{4,5}$ Even during static standing, small movements occur in the body. This postural sway is controlled by ground repulsion caused by contractions of muscles such as the gastrocnemius muscles (GCM), soleus muscles, and tibialis anterior (TA) muscles. ${ }^{6}$ Ankle joint muscles activated during the gait cycle are linked with tibialis anterior muscle activity between load response and midstance. The medial gastrocnemius (GM) and lateral gastrocnemius (GL) are activated between mid-stance and the terminal swing period. ${ }^{7}$ Furthermore, it has also been shown that stretching affects the muscle length-tension relationship and muscle strength. ${ }^{8}$ In

Copylight (C)2021 The Korean Society of Physical Therapy

This is an Open Access article distribute under the terms of the Creative Commons Attribution Non-commercial License (https:// creativecommons.org/license/by-nc/4.o.) which permits unrestricted non-commercial use, distribution, and reproduction in any medium, provided the original work is properly cited. 
particular, it was reported that continuous plantar flexion stretching for less than 15 minutes reduces muscle strength and performance due to changes in the mechanical properties of muscles and decreases centripetal biofeed biofeedback. ${ }^{9,10}$ On the other hand, it has also been reported that short static stretching for about 30 or 60 seconds did not cause muscle strength loss. ${ }^{11}$

Clinically, plantar flexor stretching is preferred to stimulate range of motion and somatosensory receptor of the ankle joint. These stretches are mainly used therapeutically in clinical practice and include dynamic, static, ballistic, and neurophysiological stretching. ${ }^{12}$ Researchers have diverse views on the biomechanical and neurological effects of stretching, but it is generally agreed that static stretching is significantly more effective than dynamic stretching due to the muscle-tendon nature of long muscles. On the other hand, dynamic stretching can cause damage as it can exceed the limits of stretchability. ${ }^{13,14}$ In addition, although researchers have various opinions on the neurological effects of stretching exercises, static stretching is a generalized stretching method whereas ballistic stretching can cause tissue damage and neurophysiological stretching requires assistance, though it increases muscle strength safely and efficiently. ${ }^{15-17}$

Temporary changes in tension and the somatosensory system due to continuous stretching of ankle joints can cause transient balance disorders. ${ }^{18-22}$ Fatigue caused by muscle activity can reduce the force required of muscles $^{23}$ and fatigue caused by stretching affects proprioception, which can diminish posture adjustment ability and disrupt joint position sensory information. Therefore, fatigue may diminish ability to maintain balance and reduce posture control ability. ${ }^{24}$ Stretching time for balance and degree of sway in the standing position immediately after static stretching of plantar flexor muscles for 5 minutes increase. ${ }^{25}$

Studies on this topic indicate that stretching negatively affects balance control, but no quantitative research has been performed to determine how much time is needed. Many studies have been conducted to improve static balance, but no consideration has been given to the activities of ankle muscles used for walking during the balance recovery period immediately after intervention. ${ }^{26-28}$ Therefore, we focused on the recovery period and by measuring the activities of TA, GM, and GL muscles while walking during recovery immediately after static stretching. We considered that increased understanding of the timings involved would reduce the risk of falls and improve health and quality of life. In particular, we sought to confirm the balance of the dominant plantar flexor and the activity of the ankle muscle while walking after static stretching for 5 minutes in a cohort of young adults.

\section{METHODS}

\section{Subjects}

This study was conducted on healthy students among students attending S University in A city. All subjects were fully explained about the purpose and method of the study before participating in the study. The subjects of this study were 2018 - to 25-year-old students who did not have a history of foot deformity, vestibular system injury, or musculoskeletal or neurological disease in the ankle or knee joints during the past 6 months (Table 1). This study was conducted with the approval of the Institutional Review Board (IRB) of S University (SM-202005-037-1). All participants were told the purpose and method of the experiment, and before starting the experiment, height and weight were measured using an automatic BMI-measuring tadiometer (BSM 370, Korea, 2011).

\section{Measurements}

\section{1) Stretch board}

All participants climbed the stretch board set at 15 to 25 degrees with their socks off and performed static stretching for 5 minutes at an angle without inconvenience to the ankle (Figure 1). ${ }^{26}$ During this period, participants were allowed to talk or use cell phones to prevent boredom. The subjects were instructed not to lean back against the wall while standing on the stretch board. Stretch board was located close to TETRAX to confirm static balance immediately after static stretching.

\section{2) Measuring stability of body (Static Balance)}

Static balance assessment was performed on the stable support surface with eyes closed using TETRAX (Sunlight Medical Ltd., Ramat Gan, Israel). The measurement method was measured during each 32 seconds static stretching for plantar flexor pre, post, post $5 \mathrm{~min}$ rest, post $10 \mathrm{~min}$ rest. Among the measured values, the stability index (ST) and weight distribution index (WDI) were used. WDI showed weight load at a percentage. The toes and heels of both feet were measured on four force plates (A-B-CD). ST showed stability by measuring the change of posture according to the change of weight, and WDI showed weight load value through per-

Table 1. General characteristics of participants.

\begin{tabular}{lc}
\hline & Subjects $(\mathrm{n}=20)$ \\
\hline Sex $(M / F)$ & $10 / 10$ \\
Age $(\mathrm{yr})$ & $21.9 \pm 1.6$ \\
Height $(\mathrm{cm})$ & $169.0 \pm 10.3$ \\
Weight $(\mathrm{kg})$ & $66.3 \pm 15.1$ \\
\hline
\end{tabular}



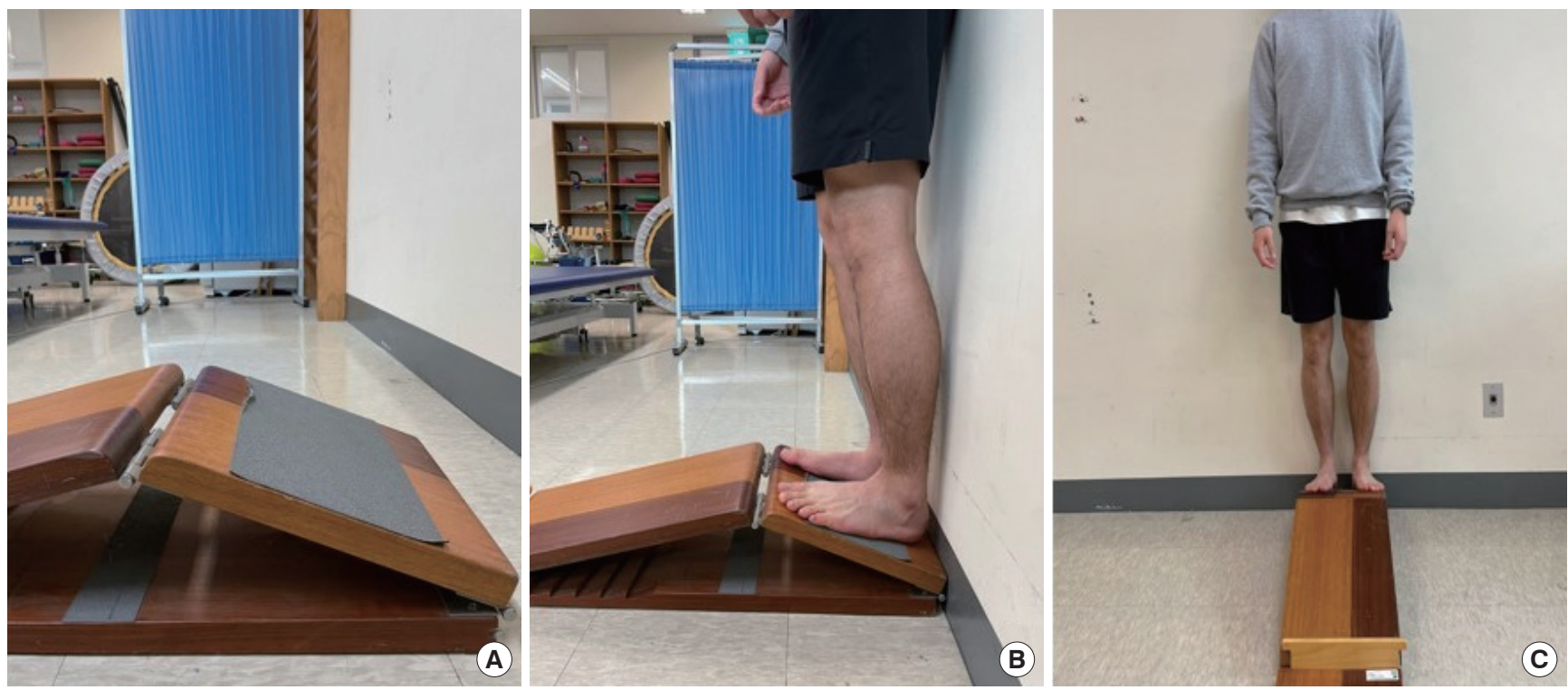

Figure 1. Stretch board. (A) Stretch board, $(B, C)$ Using the stretch board.

centage. All subjects were asked to stand in the same position and to stand in accordance with the foot shape of the foot. During the measurement, the eyes were closed to prevent visual information from being provided. The subject was asked to maintain his posture for 32 seconds. After measurement, ST and WDI were calculated and displayed on the connected computer screen. In this study, ST and WDI were used, and the lower the two values mean balance is high.

\section{3) Surface electromyography}

This study used the surface electromyography measuring equipment QUS100 (Zero wire EMG, Milan, Italy). All subjects were wearing shorts and maintaining the attachment area cleanly, and then electromyographic measurements were attached to each muscle. When measuring EMG, the spacing of the electrodes was arranged at $20 \mathrm{~mm}$. The dominant lower extremity muscles (TA, GM, GL) were measured using three channels.

As for the electrode attachment of the muscle, the TA was attached to the lateral side parallel to the middle axis of the tibia at a distance of about $1 / 4$ or $1 / 3$ between the knee and the ankle. GM and the GL were attached to the medial and lateral $2 \mathrm{~cm}$ of the midline below $2 \mathrm{~cm}$ of the knee. ${ }^{29}$ The EMG digitized signal was performed at the center of the muscle belly, and the $20-500 \mathrm{~Hz}$ bandpass filter, full wave rectification, and smoothed using root mean square (RMS). After that, the obtained muscle activity value was calculated as an RMS and averaged, and based on the muscle strength evaluation posture, it was compared and analyzed as the ratio of the MVIC.

One step was used during the gait cycle to check the changes in muscle activity of TA, GM, and GL, which are the main muscles of ankle joint movement during walking. Measurement of muscle activity was repeated three times.

\section{Procedures}

Before experiments, we measured subject heights and weights. Static balance was measured on the first day, and muscle activities on different days. Measurements were performed four times, that is, before intervention (pre), after intervention (post), 5 minutes after intervention (post 5 min), and 10 minutes after intervention (post $10 \mathrm{~min}$ ). First, static balance was measured without any intervention. The stretch board was then set to $20^{\circ}$, and static stretch was applied to plantar flexors for 5 minutes with the subject standing. Static balance was then measured at 5-minute intervals while taking a maximum rest in the sitting position. Second, measurements were carried out four times in the same manner, and muscle activities of the TA, GM, and GL muscles were measured during a step cycle rather than during static balance. Before second measurements a researcher measured maximum voluntary isometric contractions (MVICs) of the TA, GM, and GL muscles.

\section{Statistical analysis}

Statistical analysis was performed by using PASW Statistics software version 23.0 (IBM Co., Armonk, NY, USA). After confirming the normality test, one-way Repeated Measure ANOVA was performed to find out the change in balance and muscle activity according to stretching of the plan- 
tar flexor muscle (pre, post, post 5 min rest, post 10 min rest), and Fisher's LSD (Last Significant Difference) was used for post hoc analysis. Statistical significance was accepted for $\mathrm{p}$ values $<0.05$.

\section{RESULTS}

There was no significant difference in WDI pre, post, post 5 minutes rest and post 10 minutes rest $(\mathrm{p}>0.05)$. ST showed a significant difference value pre, post, post 5 minutes, and post 10 minutes $(\mathrm{p}<0.05)$. There was no significant difference in muscle activity according to pre, post, post $5 \mathrm{~min}$ utes rest, and post 10 minutes rest $(\mathrm{p}>0.05)$ (Table 2).

\section{DISCUSSION}

The purpose of this study was to investigate the acute effect of static stretching of plantar flexors for 5 minutes on static balance and ankle muscle activities when walking. After static stretching was performed on plantar flexors for 5 minutes, ST, WDI, and muscle activities of TA, GM, and GL were measured before and immediately after stretching and after rest periods of 5 and 10 minutes. A significant difference was observed between ST values, but no significant difference was observed for WDI or TA, GM, and GL activities. Furthermore, we found that after a 5 minutes rest, balance gradually recovered. Our results confirmed that static stretching of plantar flexors for 5 minutes decreased static balance and showed that at least a 5-minutes rest period is required to recover balance after stretching. Previous studies have also reported that balance ability is poor immediately after static stretching of plantar flexor muscles. ${ }^{26} \mathrm{Naga}-$ no et al. ${ }^{22}$ found that postural sway increased significantly with eyes closed after static stretching of calf muscles for 3 minutes. Han et al. ${ }^{25}$ reported that elderly showed greater posture sway immediately after static stretching of plantar flexors for 5 minutes. Based on these previous results, we performed static stretching of plantar flexors for 5 minutes with eyes closed on young adults. ST and WDI measurements confirmed a significant difference in ST. Furthermore, immediately post intervention, ST increased from $19.49 \pm 6.08$ pre-intervention to $27.97 \pm 11.00$, which suggests applying static stretch to plantar flexors for 5 minutes worsens static balance.

Static stretching of the plantar flexor for less than 15 minutes reduces muscle strength due to changes in the mechanical properties of muscles, whereas static stretching for a short time (ca. 30 to 60 seconds) does not significantly affect muscle activity or muscle strength. ${ }^{9-11}$ A significant difference was found between weight bearing rates and average electromyography findings (while walking) in a previously study that compared static and dynamic stretching. ${ }^{27}$ The shorter time than this study, two sets for 15 seconds and 15 seconds for inter-set rest, were consistent with the findings of Jung et al. ${ }^{27}$ who found that gastrocnemius muscle activity increased for a short period immediately after stretching by measuring and comparing the weight bearing rates while standing. However, this study did not deal with the results within the group, so as a result of comparing weight bearing rate according to the static stretching group, no significant difference was confirmed. Chatzopoulos et al. ${ }^{28}$ in a comparative study on the acute effects of dynamic and static stretching, showed that static stretching results in significant performance degradation in balance, agility, reaction time, and movement time. In the present study, static stretching was used, and the results obtained confirmed that balance had deteriorated. In addition, according to a study by Wallmann et al., ${ }^{30}$ which investigated the effect of static stretching of the gastrocnemius on maximum vertical jump performance, jump height was 5.6\% lower when post-stretch heights were compared with pre-stretch heights, and muscle activation of gastrocnemius was $17.9 \%$ greater for post-stretch jumps than pre-stretch jumps. These observations suggest that static stretching of the gastrocnemius negatively affected maximum jump performance despite increased muscle activity. ${ }^{30}$ In the present study, TA, GM, and GL muscle activities were confirmed in a specific walking section after static stretching of plantar

Table 2. Comparison of WDI, ST values and ankle muscle activity, according to intervention

\begin{tabular}{|c|c|c|c|c|c|c|}
\hline & pre & post & $5 \mathrm{~min}$ & $10 \mathrm{~min}$ & $\mathrm{~F}$ & $\mathrm{p}$ \\
\hline WDI (point) & $5.79 \pm 2.96$ & $7.73 \pm 3.56$ & $6.38 \pm 3.52$ & $6.66 \pm 3.12$ & 2.474 & 0.71 \\
\hline ST (point) & $19.49 \pm 6.08^{b}$ & $27.97 \pm 11.00^{a, c, d}$ & $21.26 \pm 7.90^{b}$ & $22.61 \pm 7.83^{b}$ & 8.059 & $0.001^{*}$ \\
\hline TA (\%MVIC) & $19.21 \pm 7.57$ & $19.51 \pm 8.52$ & $19.16 \pm 8.13$ & $19.24 \pm 9.77$ & 0.038 & 0.99 \\
\hline GM (\%MVIC) & $33.88 \pm 16.84$ & $39.02 \pm 24.38$ & $31.26 \pm 12.20$ & $29.26 \pm 12.87$ & 1.445 & 0.265 \\
\hline GL (\%MVIC) & $13.36 \pm 7.39$ & $14.49 \pm 7.24$ & $13.5 \pm 6.80$ & $13.51 \pm 6.34$ & 0.41 & 0.747 \\
\hline
\end{tabular}

Mean \pm SD

MVIC: Maximum voluntary isometric contraction, TA: Tibialis anterior, GM: Medial gastrocnemius, GL: Lateral gastrocnemius

astatistically different from pre, bStatistically different from post, cStatistically different from 5 minutes dStatistically different from 10 minutes.

${ }^{*} p<0.05$. 
flexors for 5 minutes, but no significant difference was found, though increased muscle activity was observed after stretching. These findings suggest static stretching increases TA, GM, and GL muscle activities but negatively affects muscle performance. Therefore, consistent with the hypothesis of this study, static stretching of plantar flexors appeared to negatively affect balance and the performance of plantar flexors. So, we think that walking immediately after stretching can increase the risk of falls because static stretching of the plantar flexor decreases balance and muscle performance.

Summarizing, the current study confirms static stretching of plantar flexors for 5 minutes significantly decreased ST and disrupted balance and recovery, but after a 5-minute rest, ST values recovered to their pre-intervention levels. Based on the results of this study, we recommend a 5-minute rest period be adopted after stretching plantar flexors before training young adults or treating patients with static balance issues.

As regards study limitations, it was our intention to recruit subjects of the same gender, but the impact of COVID-19 made this difficult and this increased the standard deviation of the weight bearing rate for stretching. Second, EMG was used to check muscle activity during walking, and we tried to control for noise as much as possible, but nevertheless, noise levels were increased by body hair and other extraneous noises. Third, though subjects practiced under experimental conditions walking patterns, individual muscle activities differed. Finally, it was difficult to control individual daily exercise routines and daily activities. Therefore, we recommend these limitations be addressed in future studies.

\section{REFERENCES}

1. Kim BG, Cho WS, Kim YS. Effects of kinesio taping on balance and gait in patients with stroke: a systematic review and meta-analysis of studies in korea. J Kor Phys Ther. 2021;33(1):21-7.

2. Lee SJ, Lee DY, Hong JH et al. The effect of kinesio taping on pain, muscle strength and balance control ability by age group: a literature review. J Kor Phys Ther. 2021;33(3):142-7.

3. Kwon JW, Yeo SS. Comparison of the static balance ability according to the subjective visual vertical in healthy adults. J Kor Phys Ther. 2020;32 (3):152-6.

4. Son SM, Kang KW. Cross-education effects of muscle strength and balance on unilateral isokinetic exercise in ankle. J Kor Phys Ther. 2020; 32(3):163-8

5. Park J. Effect of cryotherapy on muscle strength and balance on the ankle joint in patients with stroke. J Kor Phys Ther. 2021;33(2):91-6.

6. Lewis NL, Brismée JM, James CR et al. The effect of stretching on muscle responses and postural sway responses during computerized dynamic posturography in women and men. Arch Phys Med Rehabil. 2009;90(3):
454-62.

7. Moon GS. The kinematic analysis of the ankle joint and EMG analysis of the lower limbs muscle for the different walking speed. KJSB. 2005; 15(1):177-95

8. Park S. Effect of local vibration on triceps surae flexibility compared to static stretching. J Kor Phys Ther. 2020;32(4):245-9.

9. Babault N, Kouassi BY, Desbrosses K. Acute effects of 15 min static or contract-relax stretching modalities on plantar flexors neuromuscular properties. J Sci Med Sport. 2010;13(2):247-52.

10. Fowles JR, Sale DG, MacDougall JD. Reduced strength after passive stretch of the human plantarflexors. J Appl Physiol. 2000;89(3):1179-88.

11. Ogura Y, Miyahara Y, Naito H et al. Duration of static stretching influences muscle force production in hamstring muscles. J Strength Cond Res. 2007;21(3):788.

12. Sady SP, Wortman MV, Blanke D. Flexibility training: ballistic, static or proprioceptive neuromuscular facilitation? Arch Phys Med Rehabil. 1982;63(6):261-3.

13. Son DW, Shin JH. Comparison of the effects of static and dynamic stretching exercises on muscle-tendon tissue properties in gastrocnemius. Kor J Neural Rehabil. 2015;5(1):32-7.

14. Kim BR, Yi DH, Yim J. Effect of stabilizing reversal technique of proprioceptive neuromuscular facilitation and taping convergence on wrist pain and grip strength. JKCS. 2018;9(7):117-24.

15. Bradley PS, Olsen PD, Portas MD. The effect of static, ballistic, and proprioceptive neuromuscular facilitation stretching on vertical jump performance. J Strength Cond Res. 2007;21(1):223-6.

16. Kay AD, Blazevich AJ. Effect of acute static stretch on maximal muscle performance: a systematic review. Med Sci Sports Exerc. 2012;44(1):15464.

17. Perrier ET, Pavol MJ, Hoffman MA. The acute effects of a warm-up including static or dynamic stretching on countermovement jump height, reaction time, and flexibility. J Strength Cond Res. 2011;25(7):1925-31.

18. Avela J, Kyröläinen H, Komi PV. Altered reflex sensitivity after repeated and prolonged passive muscle stretching. J Appl Physiol. 1999;86(4): 1283-91.

19. Babault N, Kouassi BY, Desbrosses K. Acute effects of 15 min static or contract-relax stretching modalities on plantar flexors neuromuscular properties. J Sci Med Sport. 2010;13(2):247-52.

20. Behm DG, Bambury A, Cahill F et al. Effect of acute static stretching on force, balance, reaction time, and movement time. Med Sci Sports Exerc. 2004;36(8):1397-402.

21. Bressel E, McNair PJ. The effect of prolonged static and cyclic stretching on ankle joint stiffness, torque relaxation, and gait in people with stroke. Phys Ther. 2002;82(9):880-7.

22. Nagano A, Yoshioka S, Hay DC et al. Influence of vision and static stretch of the calf muscles on postural sway during quiet standing. Hum Mov Sci. 2006;25(3):422-34.

23. Boyas S, Haij M, Bilodeau M. Influence of ankle plantarflexor fatigue on postural sway, lower limb articular angles, and postural strategies during unipedal quiet standing. Gait Posture. 2013;37(4):547-51.

24. Suh HR, Hwang JH, Lee SY. The effect of visual information on gait parameters with induced ankle muscle fatigue. Phys Ther Rehabil Sci. 2017;6(3):140-5.

25. Han MJ, Yuk GC, Gak H et al. Acute effects of 5 min of plantar flexor 
static stretching on balance and gait in the elderly. J Phys Ther Sci. 2014; 26(1):131-3.

26. Yuk GC. The acute effects of 15 minutes plantarflexor static stretch in quite stance. Korean Soc Phys Med. 2012;7(2):191-7.

27. Jung SY, Park SS, Lee HR. The effect of stretching type on weight bearing and muscle activity in lower extremity. Journal of Sport and Leisure Studies. 2014;56(2):1009-18.

28. Chatzopoulos D, Galazoulas C, Patikas D et al. Acute effects of static and dynamic stretching on balance, agility, reaction time and movement time. J Sports Sci Med. 2014;13(2):403.

29. Criswell E. Cram's introduction to surface electromyography. London, Jones \& Bartlett Publishers, 2010:371-4.

30. Wallmann HW, Mercer JA, McWhorter JW. Surface electromyographic assessment of the effect of static stretching of the gastrocnemius on vertical jump performance. J Strength Cond Res. 2005;19(3):684. 